\section{(2) OPEN ACCESS}

\title{
Substitutions of physical activity, sedentary behaviour and sleep: associations with mental health in middle- aged and elderly persons
}

\author{
Amy Hofman, ${ }^{1}$ Trudy Voortman ๑,${ }^{1}$ M. Arfan Ikram, ${ }^{1}$ Annemarie I Luik $\oplus^{1,2}$
}

\begin{abstract}
- Additional online supplemental material is published online only. To view, please visit the journal online (http://dx.doi.org/10.1136/ jech-2020-215883).
\end{abstract}

${ }^{1}$ Department of Epidemiology, Erasmus MC University Medical Center, Rotterdam, ZuidHolland, Netherlands ${ }^{2}$ Department of Child and Adolescent Psychiatry/ Psychology, Erasmus MC University Medical Center, Rotterdam, Zuid-Holland, Netherlands

\section{Correspondence to} Dr Annemarie I Luik, Department of Epidemiology, Erasmus MC University Medical Center, Rotterdam, ZuidHolland, Netherlands; a.luik@erasmusmc.nl

Received 21 October 2020 Accepted 14 July 2021
Check for updates

(C) Author(s) (or their employer(s)) 2021. Re-use permitted under CC BY-NC. No commercial re-use. See rights and permissions. Published by BMJ.

\section{To cite: Hofman $A$,} Voortman T, Ikram MA, et al. Epidemiol Community Health Epub ahead of print: [please include Day Month Year]. doi:10.1136/jech-2020215883

\section{ABSTRACT}

Background Physical activity, sedentary behaviour and sleep are potential risk factors of mental health disorders, but previous studies have not considered the dependency between these activity domains. Therefore, we examined the associations of reallocations of time among older adults' physical activity, sedentary behaviour and sleep with depressive and anxiety symptoms using compositional isotemporal substitution analyses.

Methods We included 1943 participants (mean age 71 years, SD: 9; 52\% women) from the population-based Rotterdam Study. Between 2011 and 2016, we collected accelerometer data (mean duration 5.8 days, SD: 0.4 ) on physical activity, sedentary behaviour and sleep and selfreported data on depressive symptoms and anxiety.

Results A reallocation of 30 min more moderate-tovigorous physical activity was associated with a -0.55 (95\% Cl -1.04 to -0.06$)$ points lower depressive symptoms score when replacing sleep and a -0.59 $(95 \% \mathrm{Cl}-1.06$ to -0.12$)$ points lower score when replacing sedentary behaviour, but not when replacing light physical activity $(-0.70,95 \% \mathrm{Cl}-1.63$ to 0.24$)$. No associations were found for anxiety.

Conclusion Replacing sedentary behaviour or sleep with more moderate-to-vigorous physical activity was associated with less depressive symptoms, suggesting that mainly intensive types of physical activity are important for middle-aged and older adults in relation to depressive symptoms.

\section{BACKGROUND}

Depressive and anxiety disorders are two of the most common mental health disorders worldwide. ${ }^{1}$ These mental health disorders are associated with negative health outcomes such as reduced quality of life, more multimorbidity, higher risk of mortality ${ }^{2-4}$ and high healthcare costs, ${ }^{5}$ emphasising the importance of identifying modifiable risk factors.

Physical activity, sedentary behaviour and sleep have all been suggested as modifiable risk factors of mental health problems. Systematic reviews suggest that being more physically active is associated with a reduced risk of depressive symptoms and anxiety. ${ }^{67}$ Conversely, spending more time sedentary has been repeatedly associated with an increased risk of depressive symptoms and anxiety. ${ }^{8} 9$ Additionally, time spent sleeping has been shown to be associated with depression, with both short and long sleep duration associated with increased risk of depression, ${ }^{10}$ and its association with anxiety has also been proposed to be U-shaped. ${ }^{11} 12$
The durations of physical activity, sedentary behaviour and sleep are interrelated, as these durations sum up to the total 24 hours in a day. Therefore, an increase in any behaviour will necessarily lead to a decrease in time spent in one or more other behaviours. ${ }^{13}$ So far, studies have only taken into consideration this dependence to a limited extent. ${ }^{6-13}$

Recently, compositional isotemporal substitution analyses have been proposed to provide accurate and correct estimates by investigating the effect of substituting time spent on one activity (eg, sedentary behaviour) with time spent on another activity (eg, sleep). ${ }^{14}$ Because the activity behaviours and sleep are studied as relative values within the 24-hour composition, these analyses allow for asymmetrical results when substituting two behaviours. ${ }^{14}$ So far, this method has not been used to investigate the associations of daily activity behaviours and sleep on older adults' mental health, where all these domains have been deemed important. Therefore, it remains unclear whether, for example, an increase of sedentary time is harmful in itself, or that an association is to some extent due to a decrease in time spent physically active or asleep.

We investigated the associations between reallocations of time among older adults' daily movement behaviours-being light physical activity, moderateto-vigorous physical activity, sedentary behaviour and sleep-and depressive and anxiety symptoms, using compositional isotemporal substitution analyses. The associations were examined for the total population and stratified by sex, as moderation by sex has been suggested previously. ${ }^{15}$ We used 24-hour accelerometry to objectively examine the durations of these activity domains, to prevent bias due to self-report.

\section{METHODS}

\section{Study design and population}

This study was embedded within the Rotterdam Study, a prospective population-based cohort designed to study the occurrence and determinants of age-related diseases in the general population. ${ }^{16}$ In total, the Rotterdam Study comprises 14926 participants aged 45 years and over. The overall response rate across the three study waves was $72 \%$.

Between 2011 and 2016, 2778 unique participants were invited to wear a triaxial accelerometer for seven consecutive days and nights. A total of 2338 participants agreed to participate (84\%). Of those, 192 were excluded because of an insufficient 
amount of data (at least 4 days with minimally 1200 min per day) and 122 because of technical errors. Finally, we excluded people whose sleep diary was lacking $(n=79)$ and those without information on any of the mental health outcomes $(n=2)$, resulting in a total sample of 1943 participants.

\section{Measurements}

\section{A 24-hour activity and sleep}

Participants were asked to wear a triaxial accelerometer (GeneActiv; Activinsights, Kimbolton, Cambridgeshire, UK, http:// www.geneactiv.org/) on the non-dominant wrist for seven consecutive days and nights. Simultaneously, participants completed a sleep diary, which included questions about the times they went to bed at night and got out of bed in the morning. Accelerometers were sampled at $50 \mathrm{~Hz}$ and acceleration was expressed relative to gravity (g units; $1 \mathrm{~g}=9.81 \mathrm{~m} / \mathrm{s}^{2}$ ). ${ }^{17}$

To quantify movement acceleration, the high-pass filtered vector magnitude was computed, by applying a high-pass filter with a cut-off frequency of 0.2 Hertz to the acceleration signal, treating gravity as a low-frequency component that is filtered out. ${ }^{18}$ Accelerometer data were processed with the open access PAMPRO software, using Python (V.2.6.6). ${ }^{19}$ Non-wear time, defined as all time periods where the SD of acceleration in each of the three axes fell below $13 \mathrm{mg}$ for over 1 hour, ${ }^{18}$ was excluded from analyses. Activity was categorised into total sedentary $(<48$ $\mathrm{mg})$, light $(48<154 \mathrm{mg})$, moderate $(154<389 \mathrm{mg})$ and vigorous physical activity (>389 mg), based on a validation study in 1695 middle-aged adults. ${ }^{18}$ For this study, moderate and vigorous physical activity were combined into one category.

To compute night-time sleep duration, we used a validated algorithm. ${ }^{20}$ The algorithm uses the accelerometer data to estimate nocturnal sleep within the sleep window as reported in the sleep diary (time from going to bed to time of final waking) by detecting the prolonged periods of inactivity, defined as the absence of change in arm angle greater than $5^{\circ}$ for 5 min or more within the sleep window. ${ }^{20}$ Sleep duration was subtracted from total sedentary time to compute awake sedentary time.

\section{Depressive symptoms}

Depressive symptoms were assessed during the home interview using the Dutch version of the Center for Epidemiological Studies Depression (CES-D) scale, which was designed to assess presence and severity of depressive symptoms in the general population. ${ }^{2122}$ The sum score of the 20 questions of the CES-D ranges from 0 to 60 , with higher scores indicating more depressive symptoms. A score of 16 or higher is considered indicative for clinically relevant depressive symptoms. ${ }^{21} 22$

\section{Anxiety symptoms}

To assess anxiety symptoms, the Hospital Anxiety and Depression Scale (HADS) was completed during the home interview. ${ }^{23} 24$ The subscale for anxiety was used, which consists of seven items resulting in a total score ranging from 0 to 21. A higher score reflects more symptoms of anxiety. Scores of 8 or higher were considered as clinically relevant symptoms of anxiety. ${ }^{25}$

\section{Other variables}

Information on covariates was collected during the home interview or measured at the research centre. Self-report data were available for sex and age. Living situation was asked and categorised into 'living alone' and 'living with partner'. Educational level was categorised into primary, lower, intermediate, and higher education according to the Unesco classification.
Employment status was asked and dichotomised into 'currently employed' or 'currently not employed', based on paid employment. Body mass index (BMI) was assessed by measuring height and weight on validated scales. History of cancer, coronary heart disease, stroke and diabetes was assessed by both selfreport and continuous monitoring of medical records through digitised linkage of files from general practitioners with the study database. All events were adjudicated by a panel of study physicians. ${ }^{16}$ Continuous linkage with dispensing records from pharmacies in the study area provided drug prescription information for all study participants. For this study, psycholeptics and psychoanaleptics (Anatomical Therapeutic Chemical (ATC) classification code: N05 and N06) were examined. Smoking status was assessed during the interview and categorised into current, former and never smoker. Frequency and quantity of alcohol use was also asked during the interview and defined as the average amount of standard units per day. Finally, participants were asked during the interview whether they needed any type of walking aid to move forward.

\section{Statistical analysis}

The study population was characterised using descriptive statistics. Missing information on covariates $(<2 \%)$ was imputed using multiple imputation $(\mathrm{m}=5)$ using the mice package in $\mathrm{R}^{26}$

A daily time-use composition was constructed by using the average daily duration of four activity domains: light physical activity, moderate-to-vigorous physical activity, sedentary behaviour and sleep. This compositional variable was constructed by using the 'Compositions' package in R. ${ }^{27}$ For the construction of this variable, these activity compositions were expressed as isometric log ratio coordinates, which can be translated to the proportions spent in each activity a day. ${ }^{28}$ If a participant had enough valid data (at least 4 days with minimally $1200 \mathrm{~min}$ of data each day) but not for the full $1440 \mathrm{~min}$ a day, these proportions were adjusted in order to sum up to $1440 \mathrm{~min}(100 \%)$.

Multiple linear regression models were performed for both outcomes (depressive symptoms and anxiety), using the compositional variable as independent variable. These compositional isotemporal substitution models were used to predict the difference in the outcome variable when a fixed duration of time spent in one behaviour (eg, sedentary behaviour) was reallocated to another behaviour (eg, sleep), while the other behaviours were kept constant. ${ }^{29}$ For each of the compositions, the estimated outcome was calculated. Associations are presented as the estimated differences in the outcome between the mean composition of our study population and all possible reallocations of $30 \mathrm{~min}$. We also used logistic regression models to assess the associations of the compositions with dichotomised outcomes indicating clinically relevant depressive symptoms and clinically relevant symptoms of anxiety.

We assessed these associations in a age and sex-adjusted model and a model additionally adjusted for living situation, educational level, employment, BMI, history of cancer, coronary heart disease, stroke and diabetes, use of psycholeptics and psychoanaleptics, smoking status and alcohol intake. We assessed these associations for the total population and then stratified by sex. For sensitivity analyses, we performed the analyses excluding those who used any type of walking aid to move forward in order to assess whether the associations with mental health were potentially affected by participants that were less able to be physically active. Ad hoc, we examined for statistically significant associations whether the duration of the activity behaviours in the mean composition affected the results. Hereto, we performed 
Table 1 Study sample characteristics

\begin{tabular}{|c|c|c|c|}
\hline & Total $(\mathrm{N}=1943)$ & Men $(\mathrm{N}=940)$ & Women $(\mathrm{N}=1003)$ \\
\hline Age & $70.91(9.26)$ & $70.94(9.10)$ & $70.88(9.42)$ \\
\hline \multicolumn{4}{|l|}{ Educational level } \\
\hline Primary & $117(6.0 \%)$ & $46(4.9 \%)$ & $71(7.1 \%)$ \\
\hline Lower & $714(36.7 \%)$ & $224(23.8 \%)$ & $490(48.9 \%)$ \\
\hline Further/intermediate & $594(30.6 \%)$ & $342(36.4 \%)$ & $252(25.1 \%)$ \\
\hline Higher & $494(25.4 \%)$ & $318(33.8 \%)$ & $176(17.5 \%)$ \\
\hline \multicolumn{4}{|l|}{ Marital status } \\
\hline Living with partner & $1401(72.1 \%)$ & $797(84.8 \%)$ & $604(60.2 \%)$ \\
\hline Living alone & $542(27.9 \%)$ & $143(15.2 \%)$ & $399(39.8 \%)$ \\
\hline \multicolumn{4}{|l|}{ Occupation } \\
\hline Yes & $378(19.5 \%)$ & $207(22.0 \%)$ & $171(17.0 \%)$ \\
\hline Body mass index, $\mathrm{m} / \mathrm{kg}^{2}$ & $27.59(4.28)$ & $27.42(3.65)$ & $27.75(4.78)$ \\
\hline History of cancer & $231(11.9 \%)$ & $133(14.1 \%)$ & $98(9.8 \%)$ \\
\hline History of coronary heart disease & $177(9.1 \%)$ & $140(14.9 \%)$ & $37(3.7 \%)$ \\
\hline History of stroke & $69(3.6 \%)$ & $38(4.0 \%)$ & $31(3.1 \%)$ \\
\hline History of diabetes & $346(17.8 \%)$ & $187(19.9 \%)$ & $159(15.9 \%)$ \\
\hline Alcohol (glass/day), median (IQR) & $0.64(0.05-0.86)$ & $0.86(0.16-1.50)$ & $0.38(0.05-0.86)$ \\
\hline \multicolumn{4}{|l|}{ Smoking } \\
\hline Current smoker & $171(8.8 \%)$ & $73(7.8 \%)$ & $98(9.8 \%)$ \\
\hline Former smoker & $1141(58.7 \%)$ & $633(67.3 \%)$ & $508(50.6 \%)$ \\
\hline Never smoker & $631(32.5 \%)$ & $234(24.9 \%)$ & $397(39.6 \%)$ \\
\hline Depressive symptoms (CES-D), median (IQR) & $3.00(1.00-7.00)$ & $2.00(0.00-5.00)$ & $4.00(1.00-9.00)$ \\
\hline Anxiety (HADS), median (IQR) & $2.60(0.00-4.00)$ & $1.00(0.00-3.00)$ & $2.00(1.00-5.00)$ \\
\hline Nocturnal sleep & 6 hours $26 \mathrm{~min}(62 \mathrm{~min})$ & 6 hours $20 \mathrm{~min}(63 \mathrm{~min})$ & 6 hours $31 \mathrm{~min}(61 \mathrm{~min})$ \\
\hline Sedentary behaviour & 13 hours $32 \mathrm{~min}(79 \mathrm{~min})$ & 13 hours $49 \mathrm{~min}(78 \mathrm{~min})$ & 13 hours $18 \mathrm{~min}(76 \mathrm{~min})$ \\
\hline Light physical activity & 2 hours $28 \mathrm{~min}(32 \mathrm{~min})$ & 2 hours $24 \mathrm{~min}(31 \mathrm{~min})$ & 2 hours $32 \mathrm{~min}(31 \mathrm{~min})$ \\
\hline Moderate-to-vigorous physical activity & 1 hour $23 \mathrm{~min}(29 \mathrm{~min})$ & 1 hour $21 \mathrm{~min}(28 \mathrm{~min})$ & 1 hour $25 \min (29 \mathrm{~min})$ \\
\hline
\end{tabular}

Data are presented as mean(SD) or N(\%) unless otherwise indicated, and shown for non-imputed data. Missing data were low (<2\%).

Time spent in nocturnal sleep, sedentary behaviour, light and moderate-to-vigorous physical activity does not necessarily add up to 24 hours, as not all participants wore the accelerometer for the full 24 hours per day (participants were included when they used the accelerometer for at least 4 days with minimally 1200 min each day).

CES-D, Center for Epidemiologic Studies Depression; HADS, Hospital Anxiety and Depression scale.

the reallocations relative to different mean compositions (ie, different activity durations) with the duration set to the 25 th and 75 th percentile. Data were handled and analysed using SPSS Statistics V.24.0.0.1 (IBM) and R, V.3.6.0 (The R Foundation for Statistical Computing, Vienna, Austria).

\section{RESULTS}

Characteristics of the total study population and separated for men and women are presented in table 1 . A total of $52 \%$ of the participants were women and the mean age was 71 years (SD: 9 years). On average, men were sedentary 31 more minutes per day than women, while women spent more time sleeping than men and were also more physically active (table 1 ).

\section{Depressive symptoms}

Replacing other activities with more moderate-to-vigorous physical activity was associated with less depressive symptoms (table 2). In the adjusted model, a reallocation of 30 min more moderate-to-vigorous physical activity was associated with a -0.55 (95\% CI -1.04 to -0.06$)$ points lower depressive symptoms score when replacing sleep and a -0.59 (95\% CI -1.06 to -0.12 ) lower score when replacing sedentary behaviour, but not when replacing light physical activity $(-0.70,95 \% \mathrm{CI}-1.63$ to 0.24). Reallocations were not symmetrical; reallocations of 30 min from moderate-to-vigorous physical activity to more sleep and sedentary behaviour were associated with more depressive symptoms, but the effect sizes were larger (table 2). Results of logistic regression analyses showed similar patterns but were not statistically significant (online supplemental appendix table A).

Effect sizes were larger in men compared with women (online supplemental appendix table B). In men, a reallocation of $30 \mathrm{~min}$ more moderate-to-vigorous physical activity was associated with a -0.61 (95\% CI -1.19 to -0.02$)$ points lower depressive symptoms score when replacing sleep and a -0.66 (95\% CI -1.21 to -0.11) lower score when replacing sedentary behaviour, but not when replacing light physical activity $(-0.84,95 \%$ CI -1.93 to 0.25$)$. In comparison, in women these estimates were respectively -0.47 (95\% CI -1.26 to 0.32$),-0.45$ (95\% CI -1.22 to 0.32 ) and -0.50 (95\% CI -2.03 to 1.03$)$, and no associations were statistically significant. Similar to what we observed in the total study population, opposite reallocations, from moderateto-vigorous physical activity to sleep, sedentary behaviour and light physical activity, showed larger effect sizes.

\section{Anxiety symptoms}

Reallocations of physical activity domains were not associated with anxiety symptoms (table 3 ). When dichotomised, compositions were significantly associated with clinically relevant symptoms of anxiety in the age and sex-adjusted model, but these did not hold after further confounder adjustment (online 
Table 2 Age and sex-adjusted and confounder-adjusted results of isotemporal substitution analyses for depressive symptoms

\begin{tabular}{|c|c|c|c|c|c|c|c|c|c|}
\hline & \multicolumn{8}{|c|}{30 min more... } \\
\hline & & \multicolumn{2}{|l|}{ Sleep } & \multicolumn{2}{|c|}{ Sedentary behaviour } & \multicolumn{2}{|c|}{ Light PA } & \multicolumn{2}{|c|}{ Moderate-to-vigorous PA } \\
\hline \multicolumn{10}{|c|}{ Age and sex-adjusted model } \\
\hline \multirow[t]{4}{*}{ Replacing... } & Sleep & & & 0.08 & -0.05 to 0.21 & 0.08 & -0.36 to 0.51 & -0.73 & -1.24 to -0.22 \\
\hline & Sedentary behaviour & -0.08 & -0.20 to 0.05 & & & 0.00 & -0.44 to 0.43 & -0.81 & -1.30 to -0.32 \\
\hline & Light PA & -0.09 & -0.62 to 0.43 & -0.02 & -0.55 to 0.51 & & & -0.83 & -1.80 to 0.15 \\
\hline & Moderate-to-vigorous PA & 1.09 & 0.34 to 1.83 & 1.17 & 0.44 to 1.89 & 1.17 & 0.05 to 2.28 & & \\
\hline \multicolumn{10}{|c|}{ Confounder-adjusted model } \\
\hline & Sleep & & & 0.03 & -0.09 to 0.16 & 0.12 & -0.30 to 0.53 & -0.55 & -1.04 to -0.06 \\
\hline \multirow[t]{3}{*}{ Replacing... } & Sedentary behaviour & -0.04 & -0.16 to 0.08 & & & 0.08 & -0.33 to 0.50 & -0.59 & -1.06 to -0.12 \\
\hline & Light PA & -0.15 & -0.65 to 0.35 & -0.11 & -0.62 to 0.39 & & & -0.70 & -1.63 to 0.24 \\
\hline & Moderate-to-vigorous PA & 0.81 & 0.09 to 1.53 & 0.85 & 0.15 to 1.55 & 0.93 & -0.14 to 2.00 & & \\
\hline
\end{tabular}

Values represent the estimated differences in the outcome between the mean composition of the study population and a new composition, for example, in which 30 min more times were spent in sleep and $30 \mathrm{~min}$ less in sedentary behaviour (while light and moderate-to-vigorous physical activity were unchanged). The confounder-adjusted model was adjusted for age, sex, living situation, educational level, employment, body mass index, history of cancer, coronary heart disease, stroke and diabetes, use of psycholeptics and psychoanaleptics, smoking status and alcohol use. Bold indicates a significant association $(p<0.05)$.

PA, physical activity.

supplemental appendix table C). Results for men and women were largely similar (online supplemental appendix table D).

\section{Sensitivity analysis}

After exclusion of participants unable to move forward without any type of walking aid, results were similar for both outcomes (online supplemental appendix tables $\mathrm{E}$ and $\mathrm{F}$ ).

To assess the effect of the mean composition on the association of moderate-to-vigorous physical activity replacements with mental health, moderate-to-vigorous physical activity was also analysed with the mean composition set to the 25 th and 75 th percentile level of the population with the other activity domains proportionally distributed. The reallocations which included moderate-to-vigorous physical activity showed larger estimated differences for depressive symptoms when the level of moderate-to-vigorous physical activity in the composition was low (25th percentile). Accordingly, smaller estimated differences were found when the duration of moderate-to-vigorous physical activity in the composition was high (75th percentile) (online supplemental appendix table G). Similar patterns were seen for anxiety symptoms (online supplemental appendix table $\mathrm{H}$ ).

\section{DISCUSSION}

Our study demonstrated differential patterns of reallocations of daily activity behaviours with symptoms of depression and anxiety. Reallocations that involved moderate-to-vigorous physical activity related to depressive symptoms, while no consistent associations with anxiety were found. When clinically relevant depressive and anxiety symptoms were assessed as dichotomous outcomes, effect sizes showed similar patterns but were not significant.

More moderate-to-vigorous physical activity was associated with less depressive symptoms, in particular when it replaced sleep or sedentary behaviour. This is in line with previous work from the same cohort, concluding moderate-to-vigorous physical activities to be important factors in relation to quality of life. ${ }^{30}$ Earlier studies investigated the relationship between physical activity and depressive symptoms using traditional isotemporal substitution analysis, ${ }^{31}$ based on a similar concept but employing a different estimation procedure not taking into account the relative nature of the data, in contrast to the methods we used. ${ }^{13} 29$ Even though the employed analyses differed, our findings are in line with those of Mekary et al, who concluded that the

Table 3 Age and sex-adjusted and confounder-adjusted results of isotemporal substitution analyses for anxiety symptoms

\begin{tabular}{|c|c|c|c|c|c|c|c|c|c|}
\hline & & $30 \mathrm{~min}$ & ore... & & & & & & \\
\hline & & Sleep & & Seden & ehaviour & Light PA & & Moder & o-vigorous PA \\
\hline Age and sex-a & djusted model & & & & & & & & \\
\hline Replacing... & Sleep & & & 0.00 & -0.06 to 0.06 & 0.14 & -0.06 to 0.34 & -0.19 & -0.42 to 0.05 \\
\hline & Sedentary behaviour & 0.00 & -0.06 to 0.06 & & & 0.14 & -0.06 to 0.34 & -0.18 & -0.41 to 0.04 \\
\hline & Light PA & -0.17 & -0.41 to 0.07 & -0.17 & -0.41 to 0.07 & & & -0.35 & -0.80 to 0.09 \\
\hline & Moderate-to-vigorous PA & 0.28 & -0.07 to 0.62 & 0.27 & -0.06 to 0.61 & 0.41 & -0.10 to 0.93 & & \\
\hline Confounder-ac & ljusted model & & & & & & & & \\
\hline & Sleep & & & -0.01 & -0.07 to 0.05 & 0.16 & -0.04 to 0.35 & -0.13 & -0.36 to 0.10 \\
\hline Replacing... & Sedentary behaviour & 0.01 & -0.05 to 0.07 & & & 0.17 & -0.03 to 0.36 & -0.12 & -0.34 to 0.10 \\
\hline & Light PA & -0.19 & -0.43 to 0.04 & -0.20 & -0.44 to 0.04 & & & -0.32 & -0.76 to 0.12 \\
\hline & Moderate-to-vigorous PA & 0.19 & -0.14 to 0.53 & 0.18 & -0.15 to 0.51 & 0.35 & -0.15 to 0.86 & & \\
\hline
\end{tabular}

Values represent the estimated differences in the outcome between the mean composition of the study population and a new composition, for example, in which 30 min more times were spent in sleep and 30 min less in sedentary behaviour (while light and moderate-to-vigorous physical activity were unchanged). The confounder-adjusted model was adjusted for age, sex, living situation, educational level, employment, body mass index, history of cancer, coronary heart disease, stroke and diabetes, use of psycholeptics and psychoanaleptics, smoking status and alcohol use.

PA, physical activity 
intensity of activity is important, as self-reported brisk walking was associated with lower depression risk while walking at an average pace was not. ${ }^{32}$ However, another study using objective accelerometer data and traditional substitution models found no association between any activity domain and depressive symptoms. ${ }^{33}$ Yet overall, our results, which are the first based on the compositional isotemporal substitution model, together with previous work employing different models, suggest the importance of moderate-to-vigorous physical activity as it is associated with less depressive symptoms. Logistic regression results for clinically relevant symptoms were similar but not significant, probably due to less power in these analyses.

Another advantage of the compositional isotemporal model is that one can assess whether opposite reallocations show a symmetrical (inverse) association. ${ }^{29}$ In our findings the opposite reallocations of moderate-to-vigorous physical activity and depressive symptoms differed to some extent, that is, effect sizes were slightly smaller for substitutions including more moderateto-vigorous physical activity compared with the opposite reallocations including less moderate-to-vigorous physical activity. We might, therefore, speculate that it might be more essential for older adults to maintain their physical activity levels in order to prevent them from experiencing more depressive symptoms, than to increase their physical activity. This might be particularly relevant as some older adults will physically not be able to increase their moderate-to-vigorous physical activity. However, as our study design is cross-sectional, we cannot determine the temporality of the associations.

When the results for depressive symptoms were stratified by gender, statistically significant associations were only seen in men. Previously, a cross-sectional study concluded that men who used mental health services were significantly less active than men who did not use mental health services, while this association was not found in women. ${ }^{34}$ However, in a study among older adults, more recreational physical activity was associated with better mental health, particularly among women. ${ }^{35}$ Although results are inconsistent, our results might reflect the differences in depressive symptomatology in men and women that have been described previously. It has often been suggested that women tend to experience more of the core emotional symptoms like depressed mood and sadness, while men may experience more somatic symptoms and anger. ${ }^{36}$ We could speculate that physical activity is more directly related to the somatic symptoms of depression compared with the emotional symptoms, which might explain why we found the associations between moderate-to-vigorous physical activity and depressive symptoms in men but not in women.

In contrast to depressive symptoms, we did not find associations of replacements of activity domains with anxiety. Regarding sedentary behaviour, our results reflect the findings of Koolhaas et al, who also found no association with anxiety. ${ }^{37}$ However, based on traditional substitution models, Dillon et al concluded that a $30 \mathrm{~min}$ increase in light physical activity per day was associated with significantly lower anxiety symptoms. ${ }^{33}$ These contrasting findings may be due to differences in study population, the latter sample was recruited from a primary care setting and on average younger of age, and the differences in statistical approach. ${ }^{29}$ In a meta-meta-analysis of randomised trials in nonclinical populations a small effect of physical activity interventions on anxiety was concluded, ${ }^{6}$ yet effect of physical activity on anxiety symptoms is not consistently found in other, partly more recent, meta-analyses of clinical samples. ${ }^{38} 39$ As anxiety and depressive symptoms differ, it is also reasonable that daily activity behaviours and sleep are associated to a different extent.
Strengths of this study are the large sample size, and the application of objective accelerometer data and validated questionnaires. However, this study also has some limitations. First, as a result of the cross-sectional design, we could not establish any causality or temporality of the associations. Second, using accelerometer derived data has some limitations. For example, cycling and other sports with little activity of the arms can be underestimated, suggesting that not all physical activities can be measured accurately. ${ }^{40}$ As using these raw data from accelerometer devices is relatively new, the cut-off points that are used to categorise into intensities are not consistent. ${ }^{4041}$ Recently, it was proposed to use individual cut-off points to account for differences between individuals, ${ }^{41}$ but application in populationbased cohort studies and its feasibility needs further investigation. Third, even though we used a validated algorithm to define sleep duration which was validated against the golden standard polysomnography, it is only an estimate of sleep, which might be hard to distinguish from sedentary behaviour. In addition, our study sample included only 28 participants with a sleep duration $\geq 9$ hours, and therefore, did not allow us to assess a potentially U-shaped relationship between sleep duration and mental health. Finally, we were not able to investigate whether total duration of activities is most important itself, or that transitions or fragmentation over the day may have a more important role.

\section{CONCLUSION}

In this population-based study, more moderate-to-vigorous physical activity per day was associated with less depressive symptoms, particularly when replacing sedentary behaviour or sleep, suggesting that mainly intensive types of physical activity are related to depressive symptoms. The association pattern suggests that especially maintaining physical activity levels might be important for older adults, even when they are not increased. No associations were found between the 24-hour activity domains and anxiety symptoms. Longitudinal studies are needed to examine the temporality of these associations and potential underlying mechanisms.

\section{What is already known on this subject}

- Physical activity, sedentary behaviour and sleep have been proposed as potential modifiable risk factors of depressive and anxiety disorders.

- The durations of physical activity, sedentary behaviour and sleep are dependent on each other as they sum up to the total of 24 hours in a day, often this has not been taken into account.

- Compositional isotemporal substitution models can account for this dependency within the 24-hour day.

\section{What this study adds}

- More moderate-to-vigorous physical activity was associated with less depressive symptoms, particularly when replacing sedentary behaviour or sleep.

- No substitutions were associated with anxiety symptoms.

- This suggest that mainly intensive types of physical activity are related to depressive symptoms in a middle-aged and older population. 
Acknowledgements We acknowledge the dedication, commitment and contribution of the inhabitants, general practitioners, and pharmacists of the Ommoord district who took part in the Rotterdam Study. We acknowledge Frank van Rooij as data manager and Brenda C.T. Kieboom as study coordinator. We thank Jolande Verkroost-van Heemst for her invaluable contribution to the collection of the data.

Contributors All authors made substantial intellectual contributions to warrant coauthorship. More detailed, AH, TV and AlL designed the analysis plan, conducted the analyses and drafted the manuscript. MAI critically reviewed the analyses and manuscript. All authors approve the final version.

Funding The Rotterdam Study is funded by Erasmus Medical Centre and Erasmus University, Rotterdam, Netherlands Organisation for the Health Research and Development (ZonMw), the Research Institute for Diseases in the Elderly (RIDE), the Ministry of Education, Culture and Science, the Ministry for Health, Welfare and Sports, the European Commission (DG XII) and the Municipality of Rotterdam.

\section{Competing interests None declared.}

Patient consent for publication Not required.

Ethics approval The Rotterdam Study has been approved by the Medical Ethics Committee of the Erasmus MC (registration number MEC 02.1015) and by the Dutch Ministry of Health, Welfare and Sport (Population Screening Act WBO, licence number 1071272-159521PG). The Rotterdam Study Personal Registration Data collection is filed with the Erasmus MC Data Protection Officer under registration number EMC1712001. The Rotterdam Study has been entered into the Netherlands National Trial Register (NTR; www.trialregister.nl) and into the WHO International Clinical Trials Registry Platform (ICTRP; www.who.int/ictrp/network/primary/en/) under shared catalogue number NTR6831. All participants provided written informed consent to participate in the study and to have their information obtained from treating physicians.

Provenance and peer review Not commissioned; externally peer reviewed.

Data availability statement Data are available on reasonable request. Requests should be directed towards the management team of the Rotterdam Study ( secretariat.epi@erasmusmc.nl), which has a protocol for approving data requests. Because of restrictions based on privacy regulations and informed consent of the participants, data cannot be made freely available in a public repository.

Supplemental material This content has been supplied by the author(s). It has not been vetted by BMJ Publishing Group Limited (BMJ) and may not have been peer-reviewed. Any opinions or recommendations discussed are solely those of the author(s) and are not endorsed by BMJ. BMJ disclaims all liability and responsibility arising from any reliance placed on the content. Where the content includes any translated material, BMJ does not warrant the accuracy and reliability of the translations (including but not limited to local regulations, clinical guidelines, terminology, drug names and drug dosages), and is not responsible for any error and/or omissions arising from translation and adaptation or otherwise.

Open access This is an open access article distributed in accordance with the Creative Commons Attribution Non Commercial (CC BY-NC 4.0) license, which permits others to distribute, remix, adapt, build upon this work non-commercially, and license their derivative works on different terms, provided the original work is properly cited, appropriate credit is given, any changes made indicated, and the use is non-commercial. See: http://creativecommons.org/licenses/by-nc/4.0/.

\section{ORCID iDs}

Trudy Voortman http://orcid.org/0000-0003-2830-6813

Annemarie I Luik http://orcid.org/0000-0001-7517-197X

\section{REFERENCES}

1 Global Burden of Disease Study 2013 Collaborators. Global, regional, and national incidence, prevalence, and years lived with disability for 301 acute and chronic diseases and injuries in 188 countries, 1990-2013: a systematic analysis for the global burden of disease study 2013. Lancet 2015;386:743-800.

2 Craske MG, Stein MB, Eley TC, et al. Anxiety disorders. Nat Rev Dis Primers 2017;3:17024

3 Walker ER, McGee RE, Druss BG. Mortality in mental disorders and global disease burden implications: a systematic review and meta-analysis. JAMA Psychiatry 2015;72:334-41.

4 Read JR, Sharpe L, Modini M, et al. Multimorbidity and depression: a systematic review and meta-analysis. J Affect Disord 2017;221:36-46.

5 Mnookin S, Kleinman A, Evans T. Out of the shadows: making mental health a global development priority. Washington DC: World Bank Group, 2016.

6 Rebar AL, Stanton R, Geard D, et al. A meta-meta-analysis of the effect of physical activity on depression and anxiety in non-clinical adult populations. Health Psychol Rev 2015;9:366-78

7 Schuch FB, Vancampfort D, Firth J, et al. Physical activity and incident depression: a meta-analysis of prospective cohort studies. Am J Psychiatry 2018;175:631-48.
8 Teychenne M, Costigan SA, Parker K. The association between sedentary behaviour and risk of anxiety: a systematic review. BMC Public Health 2015:15:513.

9 Zhai L, Zhang Y, Zhang D. Sedentary behaviour and the risk of depression: a metaanalysis. Br J Sports Med 2015:49:705-9.

10 Zhai L, Zhang H, Zhang D. Sleep duration and depression among adults: a metaanalysis of prospective studies. Depress Anxiety 2015:32:664-70.

11 van den Berg JF, Luijendijk HJ, Tulen JHM, et al. Sleep in depression and anxiety disorders: a population-based study of elderly persons. J Clin Psychiatry 2009;70:1105-13.

12 van Mill JG, Hoogendijk WJG, Vogelzangs N, et al. Insomnia and sleep duration in a large cohort of patients with major depressive disorder and anxiety disorders. J Clin Psychiatry 2010;71:239-46.

13 Pedišić Z. Measurement issues and poor adjustments for physical activity and sleep undermine sedentary behaviour research - the focus should shift to the balance between sleep, sedentary behaviour, standing and activity. Kinesiology 2014:46:135-46

14 Pedišić Z, Dumuid D, Olds TS. Integrating sleep, sedentary behaviour, and physical activity research in the emerging field of time-use epidemiology: definitions, concepts, statistical methods, theoretical framework, and future directions. Kinesiology 2017:49:252-69.

15 Zhang J, Yen ST. Physical activity, gender difference, and depressive symptoms. Health Serv Res 2015;50:1550-73.

16 Ikram MA, Brusselle G, Ghanbari M, et al. Objectives, design and main findings until 2020 from the Rotterdam study. Eur J Epidemio/ 2020:35:483-517.

17 Koolhaas CM, van Rooij FJA, Schoufour JD, et al. Objective measures of activity in the elderly: distribution and associations with demographic and health factors. J Am Med Dir Assoc 2017;18:838-47.

18 White T, Westgate K, Wareham NJ, et al. Estimation of physical activity energy expenditure during free-living from wrist Accelerometry in UK adults. PLoS One 2016;11:e0167472.

19 White T. Physical activity monitor processing. Github, 2016

20 van Hees VT, Sabia S, Anderson KN, et al. A novel, open access method to assess sleep duration using a wrist-worn accelerometer. PLoS One 2015;10:e0142533.

21 Radloff LS. The CES-D scale: a self-report depression scale for research in the general population. Appl Psychol Meas 1977:1:385-401.

22 Beekman AT, Deeg DJ, Van Limbeek J, et al. Criterion validity of the center for epidemiologic studies depression scale (CES-D): results from a community-based sample of older subjects in the Netherlands. Psychol Med 1997;27:231-5.

23 Zigmond AS, Snaith RP. The hospital anxiety and depression scale. Acta Psychiatr Scand 1983:67:361-70.

24 Herrmann C. International experiences with the Hospital Anxiety and Depression Scale--a review of validation data and clinical results. J Psychosom Res 1997:42:17-41.

25 Bjelland I, Dahl AA, Haug TT, et al. The validity of the hospital anxiety and depression scale. An updated literature review. J Psychosom Res 2002;52:69-77.

26 Van Buuren S, Groothuis-Oudshoorn K. Mice: multivariate imputation by chained equations in R. J Stat Softw 2010:1-68.

27 van den Boogaart KG, Tolosana-Delgado R. "compositions": A unified R package to analyze compositional data. Comput Geosci 2008;34:320-38.

28 Mateu-Figueras G, Pawlowsky-Glahn V, Egozcue JJ. The principle of working on coordinates. Compositional data analysis: Theory and applications, 2011: 31-42.

29 Dumuid D, Stanford TE, Pedišić Ž, et al. Adiposity and the isotemporal substitution of physical activity, sedentary time and sleep among school-aged children: a compositional data analysis approach. BMC Public Health 2018:18:311.

30 Koolhaas CM, Dhana K, van Rooij FJA, et al. Physical activity types and health-related quality of life among middle-aged and elderly adults: the Rotterdam study. J Nutr Health Aging 2018:22:246-53.

31 Mekary RA, Willett WC, Hu FB, et al. Isotemporal substitution paradigm for physical activity epidemiology and weight change. Am J Epidemiol 2009:170:519-27.

32 Mekary RA, Lucas M, Pan A, et al. Isotemporal substitution analysis for physical activity, television watching, and risk of depression. Am J Epidemiol 2013;178:474-83

33 Dillon CB, McMahon E, O'Regan G, et al. Associations between physical behaviour patterns and levels of depressive symptoms, anxiety and well-being in middle-aged adults: a cross-sectional study using isotemporal substitution models. BMJ Open 2018:8:e018978.

34 Janney CA, Richardson CR, Holleman RG, et al. Gender, mental health service use and objectively measured physical activity: data from the National health and nutrition examination survey (NHANES 2003-2004). Ment Health Phys Act 2008:1:9-16.

35 Andrade-Gómez E, Martínez-Gómez D, Rodríguez-Artalejo F, et al. Sedentary behaviors, physical activity, and changes in depression and psychological distress symptoms in older adults. Depress Anxiety 2018;35:884-97.

36 Addis ME. Gender and depression in men. Clinical Psychology: Science and Practice 2008:15:153-68.

37 Koolhaas CM, van Rooij FJA, Kocevska D, et al. Objectively measured sedentary time and mental and cognitive health: cross-sectional and longitudinal associations in the Rotterdam study. Ment Health Phys Act 2019;17:100296. 
38 Bartley CA, Hay M, Bloch MH. Meta-Analysis: aerobic exercise for the treatment of anxiety disorders. Prog Neuropsychopharmacol Biol Psychiatry 2013;45:34-9.

39 Stubbs B, Vancampfort D, Rosenbaum S, et al. An examination of the anxiolytic effects of exercise for people with anxiety and stress-related disorders: a meta-analysis. Psychiatry Res 2017;249:102-8.
40 Schrack JA, Cooper R, Koster A, et al. Assessing daily physical activity in older adults: unraveling the complexity of monitors, measures, and methods. J Gerontol A Bio/ SCi Med Sci 2016;71:1039-48.

41 Rejeski WJ, Marsh AP, Brubaker PH, et al. Analysis and interpretation of Accelerometry data in older adults: the life study. J Gerontol A Biol Sci Med Sci 2016;71:521-8. 